\title{
Design of Pareto-Optimal Radar Receive Filters
}

\author{
Antonio De Maio, Marco Piezzo, Salvatore Iommelli, and Alfonso Farina
}

\begin{abstract}
This paper deals with the design of radar receive filters jointly optimized with respect to sidelobe energy and sidelobe peaks via Pareto-optimal theory. We prove that this criterion is tantamount to jointly minimizing two quadratic forms, so that the design can be analytically formulated in terms of a multi-objective optimization problem. In order to solve it, we resort to the scalarization technique, which reduces the vectorial problem into a scalar one using a Pareto weight defining the relative importance of the two objective functions. At the analysis stage, we assess the performance of the receive filters in correspondence of different values of the Pareto weight highlighting the performance compromises between the Integrated Sidelobe Level (ISL) and the Peak Sidelobe Level (PSL).
\end{abstract}

Keywords-Radar receive filter design, mismatched filter design, multi-objective optimization problem, Pareto-optimal points.

\section{INTRODUCTION}

$\mathbf{T}$ HE design of optimized low sidelobe receive filters for pulse compression radar systems is a hot research topic among the radar signal processing community since 1960 's [1], [2]. It is of fundamental interest for many radar applications including ground-based surveillance, Air Traffic Control (ATC), anti-wind shear, and radar metereology.

Some early studies can be dated back to 1967-1968 [3], [4], with reference to the IEEE journals, while to 1970 [5], [6], in the context of Russian literature. In [7], a literary survey and a selected reference list on this interesting problem is provided together with some new contributions concerning issues related to the filter length and the choice of the design criterion. According to [7], the receiving filters proposed over the years can be classified into two main categories. The former, data independent class, does not require any prior knowledge about the surrounding environment, whereas the latter, data dependent class, depends on the assumed (possibly estimated) parameters of the environment. With reference to the former class, we quote [6], [8], and [9] where the minimum Integrated Sidelobe Level (ISL) filter [6] and the minimum Peak Sidelobe Level (PSL) filter [8], [9] are respectively designed. While the minimum ISL system shares a closed form solution, the computation of the minimum PSL filter requires the solution of a Linear Programming (LP) problem [8], [9],

Effort of A. De Maio and M. Piezzo sponsored by the Air Force Office of Scientific Research, Air Force Material Command, USAF, under grant number FA8655-09-1-3006. The U.S. Government is authorized to reproduce and distribute reprints for Government purpose notwithstanding any copyright notation thereon. The views and conclusions contained herein are those of the authors and should not be interpreted as necessarily representing the official policies or endorsements, either expressed or implied, of the Air Force Office of Scientific Research or the U.S. Government.

A. De Maio, M. Piezzo, and S. Iommelli are with Universitá di Napoli "Federico II", Via Claudio 21, 80125, Naples, Italy (e-mails: \{ademaio,marco.piezzo\}@unina.it,s.iommelli@entemaxwell.it).

A. Farina is with SELEX-Sistemi Integrati, Via Tiburtina km 12.4, 00131 Rome, Italy (e-mail: afarina@selex-si.com). with reference to real optimization variables and transmitted code sequence, or the solution of a convex optimization Second Order Cone Programming (SOCP) problem [7] in the case of complex variables. Indeed, SOCP [10] problems represent a family of convex optimization programs of great interest for many signal processing applications such as beamforming [11] and target localization [12].

In this paper, we still focus on the problem of radar receive filter optimization, assuming the same signal model as in [7]. We propose a new design algorithm, based on the following criterion: joint optimization of the sidelobe energy and the peak sidelobe level. This task is tantamount to jointly minimizing quadratic forms, so that the resulting design problem can be formulated in terms of a multi-objective optimization problem. In order to solve it, we resort to the scalarization technique, where the original vectorial problem is reduced to a scalar one through the use of the Pareto-optimal theory. Thus, the proposed filters are chosen as Pareto-optimal points ${ }^{1}$ of the previously mentioned multi-objective optimization problem. The performance of the algorithm is evaluated in terms of filter response, ISL, and PSL highlighting the role played by the Pareto weight in the design procedure. Particular emphasis is given to the trade-off existing between the aforementioned metrics. Indeed, it is possible to show that a low peak sidelobe level can be swaped for a reduction of the total sidelobe energy. The trade-off is ruled by the Pareto weight, which indeed represents the parameter defining the relative importance of the two objectives in the optimization problem, namely the cost required for improving a given objective (namely, the ISL) making worse the other (namely, the PSL).

The paper is organized as follows. In Section II, we present both the signal and the receiver models; then we formulate the design problem providing the algorithm for the Pareto-optimal filter construction. In Section III, we assess the performance of the filter design scheme, also in comparison with the minimum ISL and the minimum PSL filters. Finally, conclusions are given in Section IV.

\section{A. Notation}

We adopt the notation of using boldface for vectors $\boldsymbol{a}$ and matrices $\boldsymbol{A}$. The $i$-th element of $\boldsymbol{a}$ and the $(l, m)$-th entry of $\boldsymbol{A}$ are respectively denoted by $a(i)$ and $\boldsymbol{A}(l, m)$. The transpose operator and the conjugate transpose operator are denoted by the symbols $(\cdot)^{T}$ and $(\cdot)^{H}$ respectively. The letter $j$ represents the imaginary unit (i.e. $j=\sqrt{-1}$ ). $\mathbb{C}$ is the set of real and complex numbers. For any complex number $x$, we use $\Re(x)$ and $\Im(x)$ to denote respectively the real and the imaginary part

\footnotetext{
${ }^{1}$ A Pareto-optimal solution of a multi-objective optimization problem is defined as any solution that can't be improved with respect to a component without worsening the others [13]
} 
of $x,|x|$ and $\arg (x)$ represent the modulus and the argument of $x . v^{\star}(\cdot)$ stands for the optimal value of the problem $(\cdot)$. The Euclidean norm of the vector $\boldsymbol{x}$ is denoted by $\|\boldsymbol{x}\|$. Finally, $\mathbf{0}$ denotes a zero vector or matrix as long as the size of it is clear from the context.

\section{Problem Formulation and Mismatched Filter DESIGN}

Assume that the transmitted signal is a coded pulse; denote by $M$ the number of subpulses and by $[s(1), \ldots, s(M)]^{T}$ the radar code. The waveform at the receiver end is downconverted to baseband, undergoes a subpulse matched filtering operation, and then is sampled. The vector $r=$ $[r(1), \ldots, r(P)]^{T}(P=2 L+M$, with $L$ being a design parameter) of the samples from the range cell under test can be written as [7], [14] $]^{2}$

$$
\boldsymbol{r}=\alpha_{0} \boldsymbol{s}+\sum_{n=-N+1, n \neq 0}^{N-1} \alpha_{n} \boldsymbol{J}_{n} \boldsymbol{s}+\boldsymbol{n},
$$

where $N=P-L(=L+M), \boldsymbol{s}=[\mathbf{0}, s(1), \ldots, s(M), \mathbf{0}]^{T} \in$ $\mathbb{C}^{P}$ (0 is the zero row vector of dimension $\left.L\right), \alpha_{n}$ 's are complex scalars accounting for the Radar Cross Sections (RCS's) of the range cells illuminated by the radar and for the channel propagation effects (in particular $\alpha_{0}$ refers to the RCS of the cell under test), $\boldsymbol{n}$ is the vector (assumed white) containing the filtered noise samples, and $\forall n \in\{1, \ldots, N-1\}$

$$
\boldsymbol{J}_{n}(l, m)=\left\{\begin{array}{lll}
1 & \text { if } & m-l=n \\
0 & \text { if } & m-l \neq n
\end{array} \quad(l, m) \in\{1, \ldots, P\}^{2}\right.
$$

denotes the shift matrix. Finally $\boldsymbol{J}_{-n}=\boldsymbol{J}_{n}^{T}$.

In order to estimate $\alpha_{0}$, as in [7], we focus on estimators whose analytic form is

$$
\hat{\alpha}_{0}=\frac{\boldsymbol{x}^{H} \boldsymbol{r}}{\boldsymbol{x}^{H} \boldsymbol{s}},
$$

where $\boldsymbol{x}$ is a suitable $P$-dimensional complex vector (receive filter) which can be designed according to several criteria. In particular, if $\boldsymbol{x}=s$, it is the classic matched filter to the signal $s$. Otherwise, it is usually referred to, in open literature, as mismatched filter or instrumental variable filter [7], [15].

Relevant performance metrics to optimize in the design of a receive filter are related to the energies in the sidelobes of the filter, i.e. $\frac{\left|\boldsymbol{x}^{H} \boldsymbol{J}_{n} \boldsymbol{s}\right|^{2}}{\left|\boldsymbol{x}^{H} \boldsymbol{s}\right|^{2}}, n= \pm 1, \ldots, \pm(N-1)$. Specifically, if one wants to optimize the total energy underlying the range sidelobes, it is possible to minimize the ISL $\triangleq \sum_{n=-N+1, n \neq 0}^{N-1} \frac{\left|\boldsymbol{x}^{H} \boldsymbol{J}_{n} \boldsymbol{s}\right|^{2}}{\left|\boldsymbol{x}^{H} \boldsymbol{S}\right|^{2}}$ [6], [14], so as to obtain the minimum ISL filter as an optimal solution to the optimization problem

$$
\min _{\boldsymbol{x} \in \mathbb{C}^{P}} \sum_{n=-N+1, n \neq 0}^{N-1} \frac{\left|\boldsymbol{x}^{H} \boldsymbol{J}_{n} \boldsymbol{s}\right|^{2}}{\left|\boldsymbol{x}^{H} \boldsymbol{s}\right|^{2}} .
$$

Conversely, if the main concern is to optimize the level of sidelobe peaks, the metric to be considered is the PSL $\triangleq$

\footnotetext{
${ }^{2}$ See these references for more details on the system model.
}

$\max _{n= \pm 1, \ldots, \pm(N-1)} \frac{\left|\boldsymbol{x}^{H} \boldsymbol{J}_{n} \boldsymbol{s}\right|^{2}}{\left|\boldsymbol{x}^{H} \boldsymbol{s}\right|^{2}}$. Hence, the minimum PSL filter coincides with an optimal solution to the optimization problem

$$
\min _{\boldsymbol{x} \in \mathbb{C}^{P}} \max _{n= \pm 1, \ldots, \pm(N-1)} \frac{\left|\boldsymbol{x}^{H} \boldsymbol{J}_{n} \boldsymbol{s}\right|^{2}}{\left|\boldsymbol{x}^{H} \boldsymbol{s}\right|^{2}} .
$$

Both ISL and PSL approaches are included in the more general problem of minimizing the $L_{p}$-norm of the vector containing the energies of the sidelobes. This mismatched filter design criterion is proposed in [16], where an iterative algorithm attempting to obtain an optimal solution to the problem is introduced. However, the iterative technique of [16] has no known convergence properties even if simulation results show its effectiveness in some analyzed scenarios.

Indeed, providing a filter jointly optimized with respect to the two aforementioned metrics represents an attractive task. Such a need is in part justified by the growing demand for more and more involving signal processing procedures, with particular emphasis to those capable of adapting their characteristics to different clutter features.

The idea pursued in this paper is to formulate the problem in terms of the following multi-objective optimization problem [13, pp. 174-187]:

$$
\min _{\boldsymbol{x} \in \mathbb{C}^{P}}\left(\sum_{\substack{n=-N+1 \\ n \neq 0}}^{N-1} \frac{\left|\boldsymbol{x}^{H} \boldsymbol{J}_{n} \boldsymbol{s}\right|^{2}}{\left|\boldsymbol{x}^{H} \boldsymbol{s}\right|^{2}}, \max _{\substack{n= \pm 1, \pm 2 \\ \ldots, \pm(N-1)}} \frac{\left|\boldsymbol{x}^{H} \boldsymbol{J}_{n} \boldsymbol{s}\right|^{2}}{\left|\boldsymbol{x}^{H} \boldsymbol{s}\right|^{2}}\right)
$$

where the objective is now a vector-valued function which accounts for both ISL and PSL. The main goal is to design an algorithm capable of combining a low energy profile with acceptable range sidelobe peaks. We resort to the scalarization technique in order to find filters which are Pareto-optimal solutions for (5) (more details about this topic can be found in [13], [17]). The resulting scalarized problem is proved equivalent to a convex SOCP problem which can be easily solved through interior point methods with a polynomial-time computational complexity.

\section{A. Pareto-Optimal Receive Filter Design}

This section is devoted to the design of Pareto-optimal mismatched filters; namely, we focus on filters which are Pareto-optimal solutions of problem (5). To this end, let us denote by $\boldsymbol{a}_{n}=\boldsymbol{J}_{n} \boldsymbol{s}, n= \pm 1, \ldots, \pm(N-1), \boldsymbol{a}_{0}=\boldsymbol{s}$, and

$$
\boldsymbol{A}=\left[\boldsymbol{a}_{-N+1}, \ldots, \boldsymbol{a}_{-1}, \boldsymbol{a}_{1}, \ldots, \boldsymbol{a}_{N-1}\right]^{H} \in \mathbb{C}^{(2 N-2) \times P} .
$$

$\mathrm{As}_{N-1}^{N}$ a consequence, $\quad\left|\boldsymbol{x}^{H} \boldsymbol{J}_{n} \boldsymbol{s}\right|^{2}=\left|\boldsymbol{a}_{n}^{H} \boldsymbol{x}\right|^{2}$, $\sum_{n=-N+1, n \neq 0}^{N-1}\left|\boldsymbol{x}^{H} \boldsymbol{J}_{n} \boldsymbol{s}\right|^{2}=\boldsymbol{x}^{H}\left(\boldsymbol{A}^{H} \boldsymbol{A}\right) \boldsymbol{x}$ and $\left|\boldsymbol{x}^{H} \boldsymbol{s}\right|^{2}=\left|\boldsymbol{a}_{0}^{H} \boldsymbol{x}\right|^{2}$.

In the following, we exploit the scalarization technique [13, pp. 174-187] to determine the Pareto-optimal points of the vector optimization problem (5). Precisely, let us choose any 
$\boldsymbol{\lambda} \succ_{\mathbb{R}^{2}} \mathbf{0}^{3}$, consider the scalar optimization problem

$\min _{\boldsymbol{x} \in \mathbb{C}^{P}}\left[\lambda_{1}\left(\sum_{\substack{n=-N+1 \\ n \neq 0}}^{N-1} \frac{\left|\boldsymbol{a}_{n}^{H} \boldsymbol{x}\right|^{2}}{\left|\boldsymbol{a}_{0}^{H} \boldsymbol{x}\right|^{2}}\right)+\lambda_{2}\left(\max _{\substack{n= \pm 1, \pm 2 \\ \ldots, \pm(N-1)}} \frac{\left|\boldsymbol{a}_{n}^{H} \boldsymbol{x}\right|^{2}}{\left|\boldsymbol{a}_{0}^{H} \boldsymbol{x}\right|^{2}}\right)\right]$

and let $\boldsymbol{x}$ be an optimal point. Then, according to the scalarization technique, $\boldsymbol{x}$ is a Pareto-optimal point for problem (5). The parameter $\boldsymbol{\lambda}$ represents the Pareto weight vector; namely, the vector containing the coefficients ruling the relative importance of the scalar components. The choice of the parameter $\lambda$ plays a primary role in the determination of the Pareto points; indeed, it quantifies our desire to advantage a metric with respect to the other. We explicitly notice that, setting in (6) $\lambda_{1} \neq 0$ and $\lambda_{2}=0$, we obtain the minimum ISL filter, while, if $\lambda_{1}=0$ and $\lambda_{2} \neq 0$, we come up with the minimum PSL filter. Other values of $\boldsymbol{\lambda} \succ_{\mathbb{R}^{2}} \mathbf{0}$ lead to different compromises between the ISL and the PSL.

In order to find Pareto-optimal solutions to (6), we recast problem (6) into the following problem

$$
\begin{array}{ll}
\min _{\boldsymbol{x} \in \mathbb{C}^{P}} & \lambda_{1}\left[\boldsymbol{x}^{H}\left(\boldsymbol{A}^{H} \boldsymbol{A}\right) \boldsymbol{x}\right]+ \\
& +\lambda_{2}\left[\max _{n= \pm 1, \ldots, \pm(N-1)} \boldsymbol{x}^{H}\left(\boldsymbol{a}_{n} \boldsymbol{a}_{n}^{H}\right) \boldsymbol{x}\right], \\
\text { s.t. } & \boldsymbol{x}^{H}\left(\boldsymbol{a}_{0} \boldsymbol{a}_{0}^{H}\right) \boldsymbol{x}=1
\end{array}
$$

Additionally, we observe that multiplying $\boldsymbol{x}$ for a scalar complex exponential does not affect both the constraint and the objective function; therefore, problem (7) is equivalent to

$$
\begin{array}{ll}
\min _{\boldsymbol{x} \in \mathbb{C}^{P}} & \lambda_{1}\left[\boldsymbol{x}^{H}\left(\boldsymbol{A}^{H} \boldsymbol{A}\right) \boldsymbol{x}\right]+ \\
& +\lambda_{2}\left[\max _{n= \pm 1, \ldots, \pm(N-1)} \boldsymbol{x}^{H}\left(\boldsymbol{a}_{n} \boldsymbol{a}_{n}^{H}\right) \boldsymbol{x}\right] \\
\text { s.t. } & \Re\left(\boldsymbol{a}_{0}^{H} \boldsymbol{x}\right)=1 .
\end{array}
$$

Problem (8) can be reformulated as a convex optimization problem which belongs to the class of the SOCP problems [10]. Specifically, for $\lambda_{2} \neq 0$ (if $\lambda_{2}=0$, we obtain the minimum PSL filter [8], [9]), problem (8) can be written as

$$
\begin{array}{ll}
\min _{\boldsymbol{x} \in \mathbb{C}^{P}} \max _{n= \pm 1, \ldots, \pm(N-1)} & \boldsymbol{x}^{H}\left(\gamma \boldsymbol{A}^{H} \boldsymbol{A}+\boldsymbol{a}_{n} \boldsymbol{a}_{n}^{H}\right) \boldsymbol{x} \\
\text { s.t. } & \Re\left(\boldsymbol{a}_{0}^{H} \boldsymbol{x}\right)=1,
\end{array}
$$

where $\gamma \triangleq \frac{\lambda_{1}}{\lambda_{2}}$, or equivalently as

$$
\begin{array}{ll}
\min _{\boldsymbol{x}, t} & t \\
\text { s.t. } & \left\|\boldsymbol{A}_{n} \boldsymbol{x}\right\|^{2} \leq t, n= \pm 1, \ldots, \pm(N-1), \\
& \Re\left(\boldsymbol{a}_{0}^{H} \boldsymbol{x}\right)=1, \\
& \boldsymbol{x} \in \mathbb{C}^{P}, t \in \mathbb{R},
\end{array}
$$

where $\boldsymbol{A}_{n} \triangleq\left[\begin{array}{c}\sqrt{\gamma} \boldsymbol{A} \\ \boldsymbol{a}_{n}^{H}\end{array}\right]$.

The parameter $\gamma$ can be interpreted as the weight given to the second objective (namely, the total energy under the sidelobes) with respect to the first one (namely, the peak level of the sidelobes); this clearly implies that an optimal solution to problem (8) is a function of the Pareto weight.

\footnotetext{
${ }^{3}$ We say that $\boldsymbol{\lambda} \triangleq\left(\lambda_{1}, \lambda_{2}\right) \succ_{\mathbb{R}^{2}} \mathbf{0}$ if $\lambda_{1}>0$ and $\lambda_{2}>0$
}

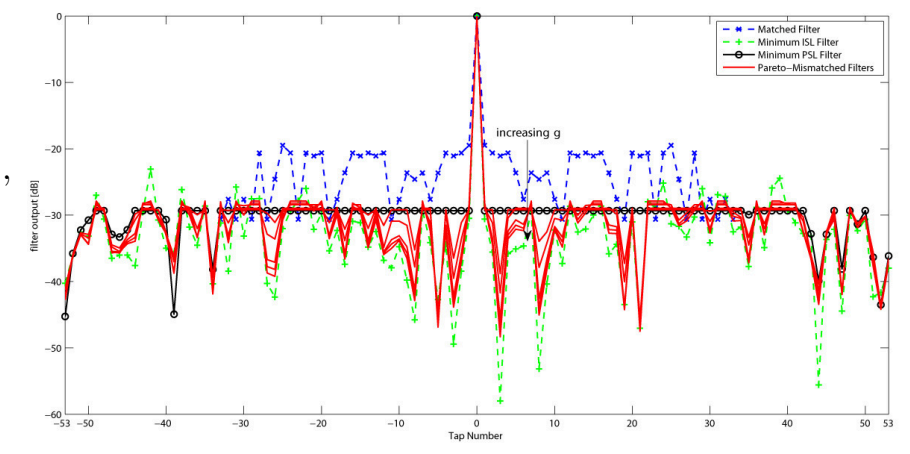

Fig. 1. Filter Output modulus versus the tap number. Minimum ISL filter (plus-dashed green curve); minimum PSL filter (circle-solid black curve); matched filter (cross-dashed blue curve); Pareto-optimal mismatched filter (9) with $\gamma \in\{0.01,0.02,0.05,0.07,0.1\}$ (solid red curves).

\section{Performance Analysis}

In this section, we assess the performance of the receive filter introduced in the previous section in terms of output modulus when the input is the transmitted sequence (zeroDoppler cut of the cross-ambiguity function), ISL, and PSL. Additionally, we provide the Pareto-optimal curve, i.e.

$$
\left\{\begin{array}{l}
\mathrm{ISL}^{\star} \triangleq \boldsymbol{x}^{\star H}(\gamma)\left(\boldsymbol{A}^{H} \boldsymbol{A}\right) \boldsymbol{x}^{\star}(\gamma), \\
\mathrm{PSL}^{\star} \triangleq \max _{n=0, \pm 1, \ldots, \pm(N-1)} \boldsymbol{x}^{\star H}(\gamma)\left(\boldsymbol{a}_{n} \boldsymbol{a}_{n}^{H}\right) \boldsymbol{x}^{\star}(\gamma),
\end{array}\right.
$$

(where ISL ${ }^{\star}$ and $\mathrm{PSL}^{\star}$ represent, respectively, the objective values of (3) and (4) in correspondence of an optimal solution $\boldsymbol{x}^{\star}(\gamma)$ to (9)); namely, the set of Pareto-optimal values, obtained through scalarization and varying the relative weight $\gamma$, for the considered optimization problem. To this end, we resort to a four-phase, length $M=34$, code with a quite low peak to sidelobe level equal to $-19.49 \mathrm{~dB}$, designed according to the method described in Appendix-A of [14]. Moreover, we use SeDuMi software [18] in our simulations to solve the SOCP problem.

In Figure 1, we show the output modulus of the receive filter in Section II-A for $P=74$ and for some values of the Pareto weight $\gamma$. In the same figure, we also plot the outputs of the minimum ISL filter, the minimum PSL filter, and the matched filter. From the plots, we can notice that the parameter $\gamma$ rules the tradeoff between ISL and PSL of the filter output. Indeed, increasing $\gamma$ we obtain filter responses which are closer and closer to the minimum ISL filter output. This is of course expected, as the greater $\gamma$, the higher the importance, in the optimization procedure, of the ISL feature with respect to the PSL one. This aspect is emphasized in Figure 2, where the related Pareto-optimal curve is plotted, as $\gamma$ ranges in the interval $[0,0.1]$. The curve is generally referred to as optimal trade-off curve, because it highlights the connection between the two objectives, $\mathrm{ISL}^{\star}$ and $\mathrm{PSL}^{\star}$, highlighting the role of the weight in the determination of their Pareto-optimal values and the cost paid for increasing one component with respect to the other. The shaded region indicates the set of all the achievable values (ISL, PSL); for example, intercepting the curve with the vertical line ISL $=\eta$ (thus considering a certain fixed value for the ISL), we can observe how big PSL has to 
TABLE I

ISL AND PSL IN dB FOR the PARETO-Optimal Mismatched Filter (9), $P=74$ AND $\gamma \in\{0.01,0.02,0.05,0.07,0.1\}$

\begin{tabular}{ccc}
\hline \hline$\gamma$ & ISL & PSL \\
\hline 0.01 & -10.017 & -29.192 \\
0.02 & -10.215 & -28.970 \\
0.05 & -10.460 & -28.440 \\
0.07 & -10.582 & -28.193 \\
0.1 & -10.593 & -27.890 \\
Matched Filter & -4.675 & -19.49 \\
Minimum ISL Filter & -10.885 & -23.064 \\
Minimum PSL Filter & -9.610 & -29.330 \\
\hline \hline
\end{tabular}

be in order to achieve ISL $=\eta$. The same interpretation arises intercepting the curve with an horizontal line $\mathrm{PSL}=\beta$ (thus considering a certain fixed value for the PSL), which makes evident the minimum achievable value ISL in order to ensure $\mathrm{PSL}=\beta$. The slope of the optimal trade-off curve at a Paretooptimal value shows the local optimal trade-off between the two objectives; steep slopes lead to large variations of ISL ${ }^{\star}$ in correspondence of small changes in $\mathrm{PSL}^{\star}$ (this is actually what happens in the lower right region of the curves in Figure 2).

In Table I, we explicitly report the tradeoff between ISL and PSL; as already pointed out, increasing $\gamma$ is tantamount of getting lower and lower PSL values, at the price of higher and higher ISL levels.

In Figures 3a-3b, we analyze the behavior of the ISL and PSL (still for the filter designed according to the criterion of Section II-A) versus the parameter $L$, which rules the length of the filter. For comparison, in the same figures, we also report the behavior of the minimum ISL filter, the minimum PSL filter, and the matched filter. The plots confirm that the longer the filter, the lower the corresponding ISL and PSL values. Indeed, this result is expected, since increasing $L$ is tantamount to providing more degrees of freedom to the filter optimization process.

Finally, in Figures $4 a-4 b$, we analyze the Doppler tolerance of the filters shown in Figure 1. Specifically, we assess the degradation of the actual ISL and PSL due to the presence of a Doppler shift in the useful signal. The curves in the figures, representing either ISL or PSL versus the normalized Doppler frequency $\nu_{d}$, ranging in the interval $\left[-\Delta_{\nu}, \Delta_{\nu}\right]$, highlight that

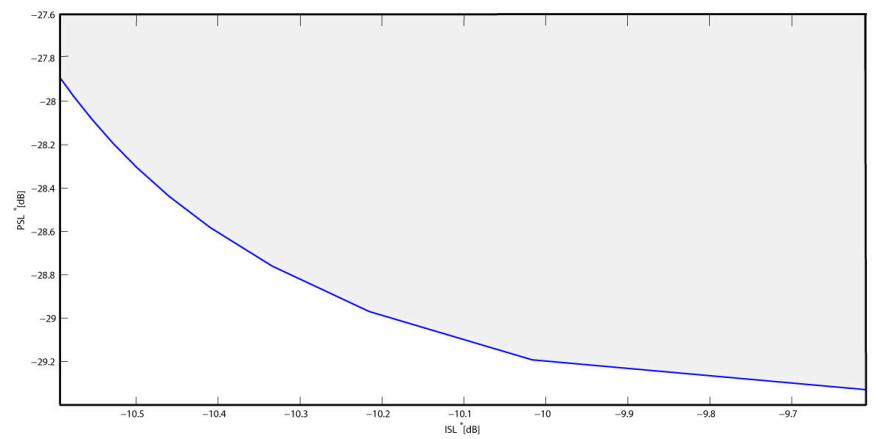

Fig. 2. Pareto-optimal curve for $\gamma \in[0,0.1]$, with a four-phase code of length $M=34$. The set of achievable values above the curve is shaded in gray.

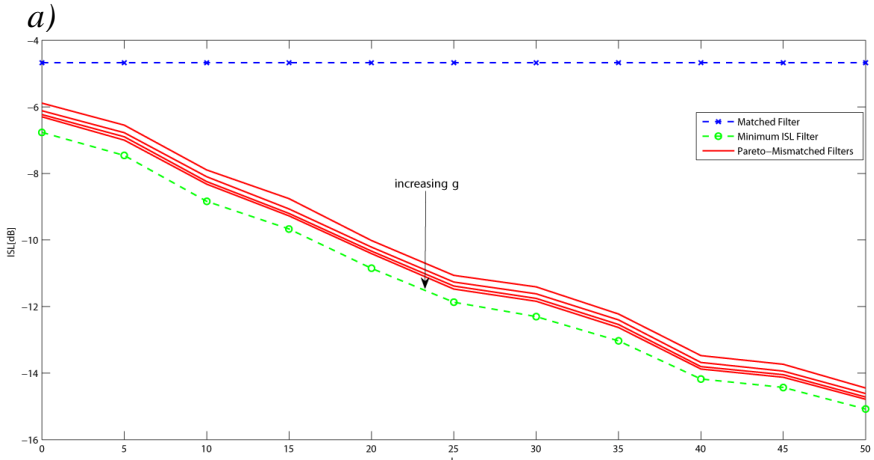

b)

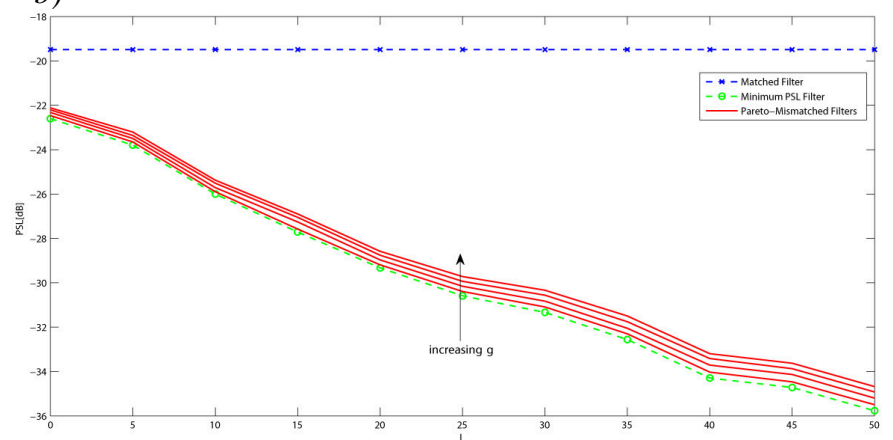

Fig. 3. a) ISL versus $L$ for the Filters in Figure 1, b) PSL versus $L$ for the Filters in Figure 1.

$L=[0,5,10,15,20,25,30,35,40,45,50]$. Matched-Filter (blu x-dashed curve). Minimum ISL filter (green circle-dashed curve). Pareto-optimal mismatched filters (red-solid curves).

the higher the target Doppler (i.e. the discrepancy from the nominal condition), the worse the ISL and PSL associated with the filter (for all the considered values of the parameter $\gamma$ ). Nevertheless, for the simulated shift values, the new filters still guarantee a performance level which is superior than that ensured by the matched filter, both in terms of ISL and PSL.

\section{CONCLUSIONS}

In this paper, we have considered the design of radar receive filters according to the following criterion: joint optimization of the ISL and PSL performance metrics. The problem has been formulated in terms of a multi-objective optimization problem. In order to solve it, we have resorted to the scalarization technique, thus focusing on the solutions which are Pareto-optimal for the aforementioned problem. At the analysis stage, we have assessed the performance of the considered receive systems providing filter responses and highlighting the tradeoff between ISL and PSL. Moreover, we have studied the Pareto-optimal curve, showing the effects of the Pareto weight on the performance trade-off. Finally, we have analyzed the Doppler tolerance associated with the considered receive systems.

Possible future developments might be focused on the comparison between the proposed design criterion and that based on the $L_{p}$ norm minimization of the filter sidelobe energies. Additionally, it might be of interest the study of quantization effects on the filter coefficients as well as of the possible imbalance between the I and Q channels of the processing chain. 
a)

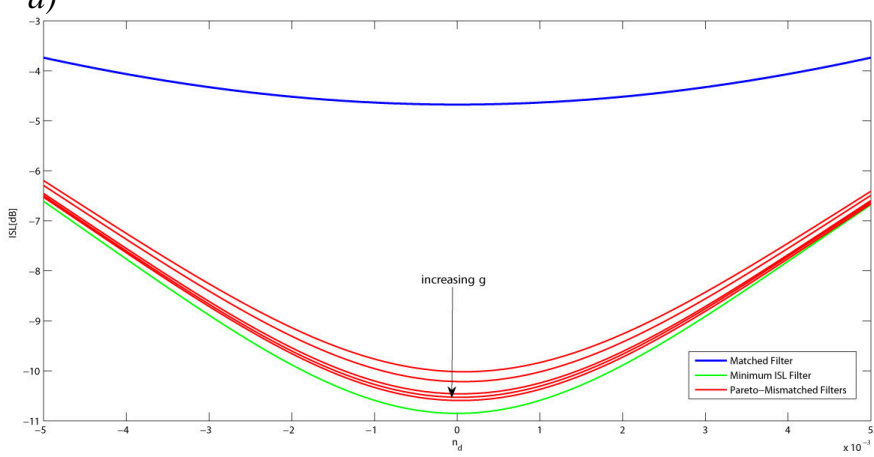

b)

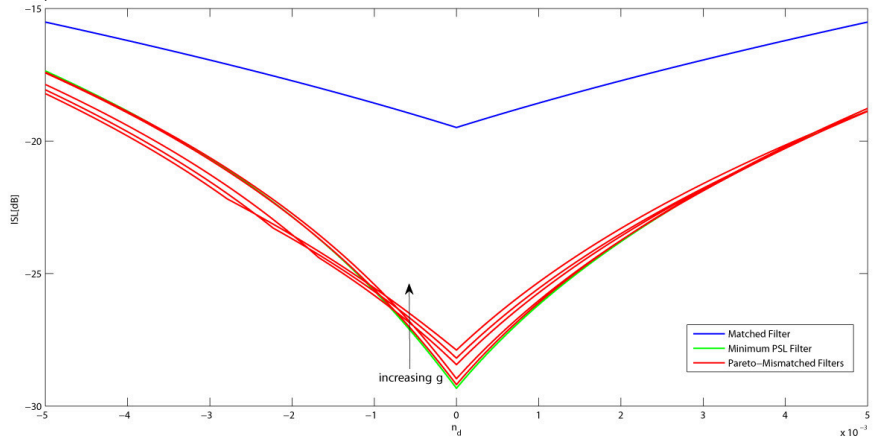

Fig. 4. a) ISL versus $\nu_{d}$ for the Filters in Figure 1, b) PSL versus $\nu_{d}$ for the Filters in Figure 1.

$\Delta_{\nu}=0.005$. Matched Filter (blue-solid curve). Minimum ISL Filter (greensolid curve). Pareto-optimal Mismatched Filters (red-solid curves).

\section{REFERENCES}

[1] M. I. Skolnik, Introduction to radar systems, 3rd ed. Mc Graw Hill, 2001.

[2] C. E. Cook and M. Bernfield, Radar signals: nn introduction to theory and application. New York: Academic Press, 1967.

[3] D. F. DeLong and E. M. Hofstetter, "On the design of optimum radar waveforms for clutter rejection," IEEE Transactions on Information Theory, vol. 13, no. 3, pp. 454-463, July 1967.
[4] C. Stutt and L. Spafford, "A best mismatched filter response for radar clutter discrimination," IEEE Transactions on Information Theory, vol. 14, no. 2, pp. 280-287, March 1968.

[5] Y. I. Abramovich and M. B. Sverdlik, "Synthesis of a filter which maximizes the signal-to-noise radio under additional quadratic constraints," Radio Engineering and Electronic Physics, vol. 15, no. 11, pp. 19771984, 1970

[6] V. T. Dolgochub and M. B. Sverdlik, "Generalized $\gamma$-filters," Radio Engineering and Electronic Physics, vol. 15, pp. 147-150, January 1970

[7] P. Stoica, J. Li, and M. Xue, "Transmit codes and receive filters for radar," IEEE Signal Processing Magazine, vol. 25, no. 6, pp. 94-109, November 2008.

[8] Y. I. Abramovich and M. B. Sverdlik, "Synthesis of filters maximizing the signalto- noise ratio in the case of a minimax constraint on the sidelobes of the crossambiguity function," Radio Engineering and Electronic Physics, vol. 16, pp. 253-258, February 1971.

[9] S. Zoraster, "Minimum peak range sidelobe filters for binary phasecoded waveforms," IEEE Transactions on Aerospace and Electronic Systems, vol. 16, no. 1, pp. 112-115, January 1980.

[10] A. Nemirovski, Lectures on modern convex optimization. Class Notes, Georgia Institute of Technology, Fall 2005

[11] S. A. Vorobyov, A. B. Gershman, and Z.-Q. Luo, "Robust adaptive beamforming using worst-case performance optimization: a solution to the signal mismatch problem," IEEE Transactions on Signal Processing, vol. 51, no. 2, pp. 313-324, February 2003.

[12] K. Yang, G. Wang, and Z.-Q. Luo, "Efficient convex relaxation methods for robust target localization by a sensor network using time differences of arrivals," IEEE Transactions on Signal Processing, vol. 57, no. 7, pp. 2775-2784, July 2009.

[13] S. Boyd and L. Vandenberghe, Convex Optimization. Cambridge University Press, 2003.

[14] K. Griep, J. A. Ritcey, and J. J. Burlingame, "Poly-phase codes and optimal filters for multiple user ranging," IEEE Transactions on Aerospace and Electronic Systems, vol. 31, no. 2, pp. 752-767, April 1995.

[15] P. Stoica, J. Li, and M. Xue, "On binary probing signals and instrumental variables receivers for radar," IEEE Transactions on Information Theory, vol. 54, no. 8, pp. 3820-3825, August 2008.

[16] J. E. Cilliers and J. C. Smit, "Pulse compression sidelobe reduction by minimization of $L_{p}$-norms," IEEE Transactions on Aerospace and Electronic Systems, vol. 43, no. 3, pp. 1238-1247, July 2007.

[17] K. Deb, Multi-objective optimization using evolutionary algorithms, 1st ed. John Wiley \& Sons, June 2001.

[18] J. F. Sturm, "Using SeDuMi 1.02, a MATLAB toolbox for optimization over symmetric cones," Optimization Methods and Software, vol. 11-12, pp. 625-653, August 1999. 